\section{ICAM'97/E-MRS'97 to Be Held in Strasbourg, France}

The International Conference on Advanced Materials (ICAM'97) and the European Materials Research Society Spring Meeting (E-MRS'97) will be held in Strasbourg, France on June 16-20, 1997 at Congress Center. The Industrial Exhibition is scheduled for June 18 and 19. E-MRS conference chairs are G.M. Crean (Ireland) and P. Glasow (Germany). E-MRS, consisting of materials scientists, engineers, physicists, and chemists worldwide, will present the latest scientific and technical reports in the field of materials. The conference includes 13 symposia running in parallel with a balance from fundamental and applied research, development, and technology with medium- and short-term perspectives. ICAM'97, organized by the International Union of Materials Research Societies, will offer a forum on the latest development in the field of advanced materials worldwide.

Proceedings will be published by Elsevier/North Holland. The conference language is English.
Following is a list of symposia titles:

- Fullerenes and Carbon-Based Materials (Symposium A);

- Epitaxial Thin-Film Growth and Nanostructures (B);

- Recent Developments in Electron Microscopy and X-Ray Diffraction of Thin-Film Structures (C);

- Computational Modeling of Issues in Materials Science (D);

- Material Aspects for Electric Vehicles (E);

- Advanced Materials Education (F);

- Biomaterials: Perspectives for Research and Industry at the Century Change $(\mathrm{G})$;

- Biodegradable Polymers and Macromolecules $(\mathrm{H})$;

- Interrelation of Science Economy and Policy in Materials Research and Processing (I);

- Light-Weight Materials in Transportation (J);

- Coatings and Surface Modifications for Surface Protection and Tribological Applications (K):

- III-V Nitrides Semiconductors and
Ceramics: From Material Growth to Device Applications (L); and

- Materials, Physics, and Devices for Molecular Electronics and Photonics (M).

Registration fees, to be paid in french francs, are 2500,00 FF before May 20, 1997 and 2800,00 FF after May 20, 1997, which cover the proceedings of one symposium, breaks, lunches, E-MRS membership for one year, and 12 issues of MRS Bulletin; and 1300,00 FF before May 20 and 1600,00 FF after May 20 for students, which include breaks and lunches. The conference secretariat is P. Siffert, E-MRS 1997 Spring Meeting, BP 20, 67037 Strasbourg Cedex 2 , France; 33-03-88-10-65-43; fax 33-03-88-1062-93; e-mail EMRS@FRCPN11.IN2P3.FR.

Companies interested in exhibiting may contact Equipment Exhibition at the address and numbers given above. For Visa assistance, contact Visa Assistance at the street and e-mail addresses above or fax 33-03-88-10-63-43.

\section{Advanced Chemicals \\ \& Materials Processing} -

- Electronic Grade Red Phosphorus

- GaN Powder \& submillimeter size single crystals

- InP polycrystalline charge, single crystals, wafers

- Bulk growth of III-V compound crystals with custom doping \& orientation

- R\&D Consulting Services for the Electronics Materials Industry

- Outsource and/or subcontract R\&D and manufacturing services

- Materials Purification

- Custom Single Crystals

- Custom Equipment Design

Visit us at Booth 319 at the Spring MRS Exhiblt, April 1-3, 1997

\section{Parke Mathematical Laboratories 450 Chelmsford Street Lowell, MA 01851 (508) 934-0854 FAX (508) 934-0731 E-mall: pml-parkemath.com Wob Slto: http://www.parkemath.com}

Circle No. 12 on Reader Service Card.

\section{ATTENTION STUDENTS!}

\section{Announcing a Materials Photo Contest}

Materials Research Society University Chapters and student teams are invited to participate in a Symmetry Photo Competition at the 1997 MRS Spring Meeting in San Francisco. Entries will be displayed in San Francisco and prizes awarded in several categories. For more information, see the MRS Website (http:/ / www.mrs.org/ sections/contest) or contact Beth Stadler, chair of the MRS Academic Affairs Committee (617-491-3762; stadler@maxwell.rl.plh.af.mil). Entries must be received no later than March 15, 1997.
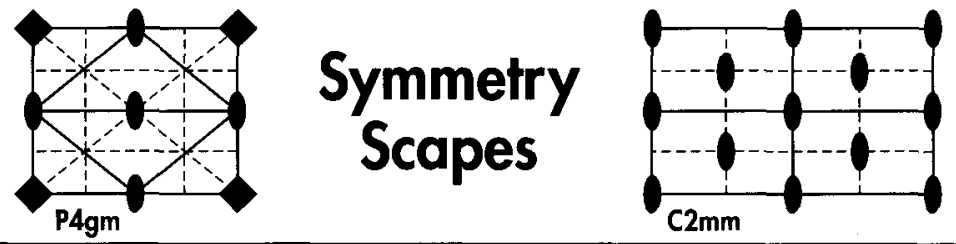